Check for updates

Cite this: Phys. Chem. Chem. Phys., 2021, 23, 9851

Received 21st February 2021, Accepted 26th March 2021

DOI: 10.1039/d1cp00807b

rsc.li/pccp

\title{
Single-scan measurements of nuclear spin singlet order decay rates
}

\author{
Giulia Melchiorre, ${ }^{a}$ Ciara Nelder, ${ }^{a}$ Lynda J. Brown, ${ }^{a}$ Jean-Nicolas Dumez (D) ${ }^{b}$ and \\ Giuseppe Pileio (D) *a
}

\begin{abstract}
Measurements of singlet spin order decay rates are time consuming due to the long-lived nature of this form of order and the typical pseudo-2D mode of acquisition. Additionally, this acquisition modality is not ideal for experiments run on hyperpolarized order because of the single-shot nature of hyperpolarization techniques. We present a methodology based on spatial encoding that not only significantly reduces the duration of these experiments but also confers compatibility using spin hyperpolarization techniques. The method condenses in a single shot the variable delay array used to measure decay rates in conventional pseudo-2D relaxation experiments. This results in a substantial time saving factor and, more importantly, makes the experiment compatible with hyperpolarization techniques since only a single hyperpolarized sample is required. Furthermore, the presented method, besides offering savings on time and costs, avoids reproducibility concerns associated with repetition in the hyperpolarization procedure. The method accelerates the measurement and characterization of singlet order decay times, and, when coupled with hyperpolarization techniques, can facilitate the quest for systems with very long decay times.
\end{abstract}

\section{Introduction}

NMR methodologies that exploit various aspects of long-lived nuclear spin singlet order - all groupable under the name singlet NMR - are under constant development. ${ }^{1-17}$ The three main properties of this form of spin order - NMR silent, accessible on demand and long-lived - have found a variety of applications including: quantification, with enhanced sensitivity, of ligand binding ${ }^{18,19}$ measurements of very slow translational dynamics; ${ }^{20-25}$ and their exploitation as molecular tags that can preserve information over a long time. ${ }^{26-36}$ Long-lived spin order is exploited as a vehicle to preserve spin hyperpolarization gained through techniques such as PHIP, ${ }^{37}$ SABRE $^{38}$ and dissolution-DNP. ${ }^{39}$ The quest for a biocompatible molecular marker that supports hyperpolarized long-lived spin order to be used for in vivo imaging applications is still open and has the potential to bring about important new possibilities in MRI. ${ }^{40}$

At the core of all these developments is the measurement of the singlet order decay constant $\left(T_{\mathrm{S}}\right)$ and its theoretical modelling. ${ }^{41}$ The intelligent design of molecules bearing singlet states has led to molecules displaying singlet order lifetimes of the order of hours. ${ }^{42,43}$ However, the procedure to interrogate whether a system displays such long lifetimes is quite tedious

\footnotetext{
${ }^{a}$ School of Chemistry, University of Southampton, SO17 1BJ, Southampton, UK. E-mail: g.pileio@soton.ac.uk

${ }^{b}$ CEISAM, CNRS, Université de Nantes, 44300 Nantes, France
}

and costly in terms of money and time: a molecular system is devised on paper according to actual knowledge and numerical/ analytical calculations; a synthetic route is designed (often involving multiple isotopic labeling) and the molecule synthesized before its relaxation properties are characterized. Understanding of the interplay of the different relaxation mechanisms in the molecule is then fed back into the molecule design and, often, the synthesis of a new derivative is required, which adds to time and costs. Such processes would be enormously facilitated (in terms of both time and costs) if one could measure the singlet lifetime on naturally abundant isotopic spin pairs by using hyperpolarization techniques to reveal the signal in such lowsensitivity species. However, although hyperpolarization is able to provide signals from singlet order in natural abundant systems, measuring their lifetime is not so straightforward.

The conventional method of measuring singlet decay constants is based on the following steps: (1) create longitudinal order either by waiting for the sample to return to equilibrium in a magnetic field or by hyperpolarizing the sample with one of the cited techniques (the duration of this step is indicated as $\tau_{0}$ ); (2) convert longitudinal order into singlet order ${ }^{8}$ (depending on the technique this is of the order of tens to hundreds of milliseconds, thus negligible in this context); (3) wait for an incremental variable time delay $\left(\tau_{i}\right)$ during which the singlet order gradually decays; (4) reconvert the remaining singlet order into transverse magnetization and acquire the signal (again of negligible duration in this context); (5) repeat all four previous 
steps nr-times (most typically 8 or 16 ) each time using a longer value for the variable delay $\tau_{i}$. In the case of a low signal-tonoise ratio, steps 1 to 5 are also repeated ns times. The total duration of such an experiment is

$$
\mathbb{T} \cong \mathrm{ns}\left(\tau_{0} \mathrm{nr}+\sum_{i=1}^{\mathrm{nr}} \tau_{i}\right)
$$

neglecting the duration of the pulse sequence blocks to prepare and reconvert the singlet order and the acquisition time.

For example, to measure a $T_{\mathrm{S}}$ estimated to be of the order of $60 \mathrm{~min}$, one would wait for, at least, $\tau_{0} \cong 3 T_{\mathrm{S}}=180 \mathrm{~min}$ and use a variable delay array going from 0 to $180 \mathrm{~min}$ in 7 spaced points (to make it simple), i.e. $\mathrm{nr}=7$ and $\tau_{i}=[0,30,60,90,120$, 150, 180] $\mathrm{min}$; under these conditions the total experimental time would result in $\mathbb{T}>32 \mathrm{~h}$ per scan $(\mathrm{ns}=1)$. This time can be reduced to $\sim 11 \mathrm{~h}$ if the singlet order is destroyed ${ }^{6}$ at each of the $\mathrm{nr}$ repetitions such that $\tau_{0}$ could be kept of the order of $3 T_{1}$ $\left(T_{1}\right.$ is assumed $30 \mathrm{~s}$ in this example) rather than $3 T_{\mathrm{S}}$. Experiments to measure $T_{\mathrm{S}}$ in low-gamma singlet spin pairs can therefore take several days because of the combined need for taking many transients and the many minutes long typical $T_{\mathrm{S}}$ values.

Sensitivity can be greatly enhanced using hyperpolarized spin order. However, even in this case the measurements of singlet decay rates are complicated by the need to freshly prepare hyperpolarized order at each of the $\mathrm{nr}$ repetitions and this, depending on the hyperpolarization technique, is a costly and time-consuming procedure, often presenting issues of reproducibility. In dissolution-DNP, for example, the preparation of hyperpolarized order typically ranges between $10 \mathrm{~min}$ and $1 \mathrm{~h}$, therefore at each $\mathrm{nr}$ repetition $\tau_{0}$ can be 10-60 min long. Methodology to circumvent these issues in hyperpolarized experiments has been published. The most basic method emulates the use of small-flip-angle pulses used in measuring $T_{1}$ on hyperpolarized samples. For instance, a reduced efficiency singlet-to-magnetization step (such as, for example, spin-lock induced crossing (SLIC) or chemical shift scaling (CSS)) can be used to convert only a small amount of the hyperpolarized singlet pool into observable magnetization as demonstrated in long-lived hyperpolarization obtained with PHIP. ${ }^{44}$ However, because the spin order lost during each conversion step is not known, multiple experiments with variable repetition times are needed to access the value of $T_{\mathrm{S}}$. Also, only a fraction of the initial batch of hyperpolarized signal is used at each observation. In order to take advantage of the full hyperpolarized signal, a recycling scheme ${ }^{45}$ has been proposed. This scheme runs as follows: a batch of hyperpolarized longitudinal order is repeatedly converted into long-lived order, stored for a variable time, reconverted into transverse magnetization, read and, immediately after, re-stored in the form of long-lived order to be used later for the next point in the variable delay array. Both these methods solve costs and reproducibility issues at once and reduce the total experimental time by $\tau_{0}(\mathrm{nr}-1)$ per scan since a single batch of hyperpolarized order is used. The disadvantage of the recycling procedure is that experimental losses are observed at each singlet-to-magnetization-to-singlet conversion and this appears as an extra contribution to the decay time that must be characterized and accounted for.

Spatial parallelization is a powerful approach to accelerate NMR experiments that require a stepwise incrementation of a delay. It has been applied, for example, to the evolution delay of $2 \mathrm{D}$ NMR experiments, ${ }^{46}$ the recovery delay in relaxation experiments, ${ }^{47}$ and the duration of the diffusion encoding gradient in diffusion experiments. ${ }^{48}$ Spatial parallelization is especially useful in combination with single-shot hyperpolarization methods and has notably been combined with dissolution dynamic nuclear polarisation. $^{49-53}$

In this paper, we introduce a methodology that uses spatially selective long-lived spin order preparation techniques ${ }^{23}$ to reduce the experimental time required to measure $T_{\mathrm{S}}$ to a duration equivalent to the longest point in the variable delay array: $\mathbb{T} \sim \tau_{\mathrm{nr}}$. This procedure is valuable in further cutting down on experimental time (and associated costs) and, as the other existing methods, it would only require a single hyperpolarized sample thus reducing preparation time, costs and irreproducibility issues.

\section{Experimental}

\section{Proposed methodology}

The methodology presented in this paper is based on the use of a space-selective magnetization to singlet conversion schemes (sM2S/sS2M $)^{23}$ and can be implemented in two different ways which mix together either a single non-selective M2S and a train of sS2M (v1) or a train of sM2S and a single non-selective S2M (v2). Both variants achieve the same goal of shortening the measurement time, but only the first variant is compatible with hyperpolarized NMR. This is because in v2 the hyperpolarized spin order is stored as "short-lived", longitudinal magnetization during the sM2S train. In v1 a non-selective M2S is run to convert thermal or hyperpolarized longitudinal order into singlet order across the whole sample. Then, a train of sS2M blocks followed by a relaxation delay and acquisition is run, repeatedly, to convert singlet order back to transverse order only in selected portions (slices) of the sample. At each run, the sS2M is instructed to address a different portion of the sample so that the singlet order from different portions is converted, and the associated signal acquired, at different times. Although the acquisition of a conventional FID is possible, we have here preferred to acquire the signal through a $1 \mathrm{D}$ spin echo imaging sequence so that the thickness and position of the slices addressed is clearer and under control. Using this methodology, singlet order is prepared in all slices simultaneously; however, the time elapsed from preparation to detection varies for the different slices, so the whole array of the variable delay time can be encoded in a single run. Singlet order is insensitive to gradient pulses, therefore, once this form of order is prepared in a given slice all successive gradients used to address a different slice have no deleterious effects. We have dubbed this general methodology as single-scan- $T_{\mathrm{S}}$ (SSTS). The pulse sequence for its 


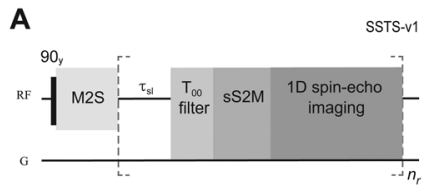

B

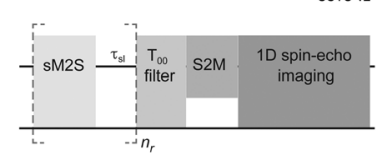

C

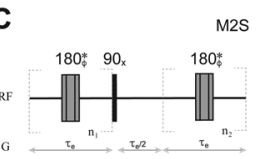

D

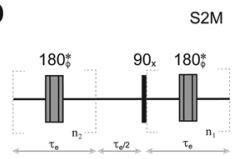

E $90_{y} 90_{\beta_{m}} 90_{x}$

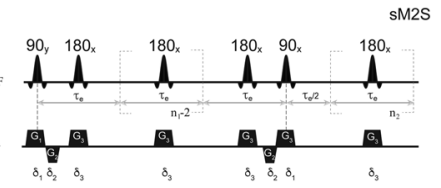

H

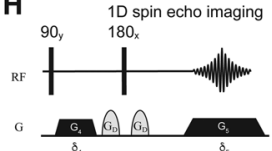

G

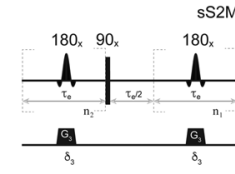

I

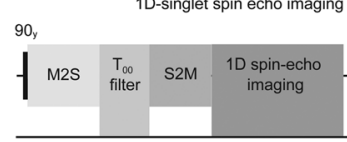

Fig. 1 (A and B) SSTS pulse sequences proposed in this work with details of the M2S (C), S2M (D). Too filter (E), sM2S (F) SS2M (G) and 1D spin echo imaging $(H)$. The sequence for singlet-order-filtered $1 D$ spin echo imaging is presented in (I). $n_{1}=\pi J /(2 \Delta \nu)$ and $n_{2}=n_{1} / 2 \cdot \tau_{\mathrm{e}}=1 /\left(2 \sqrt{\left(J^{2}+\Delta \nu^{2}\right)}\right)$. $\beta_{\mathrm{m}}$ indicates the magic angle and * indicates a composite $180^{\circ}$ pulse built as $90_{x} 180_{y} 90_{x}$ and with overall phase $\phi$ cycled within each echo train as $[x, x, \bar{x}, \bar{x}, \bar{x}, x, x, \bar{x}, \bar{x}, \bar{x}, x, x, x, \bar{x}, \bar{x}, x]$.

first implementation (SSTS-v1) is illustrated in Fig. 1A; its different steps can be summarized as follows:

(1) A M2S ${ }^{54,55}$ block (Fig. 1C) creates long-lived singlet order at the same time across the whole sample.

(2) The singlet order is allowed to decay for a time $\tau_{\mathrm{sl}}$ (kept constant or varied at each repetition).

(3) A $T_{00}$ filter $^{56}$ (Fig. 1E) is applied to filter out possible M2S by-products different from singlet order.

(4) A sS2M block (Fig. 1G) converts singlet order back to transverse magnetization only in a selected region of space (an axial slice perpendicular to the tube long axis in the examples below). The orientation, position and thickness of this slice depend on the axis along which the gradients $G_{3}$ is applied, its intensity and the offset and bandwidth of the shaped 180 degrees pulses (details below).

(5) A 1D spin echo imaging sequence (Fig. 1H) is run to yield the sample profile along the direction selected by the gradients $\mathrm{G}_{4}$ and $\mathrm{G}_{5}$.

(6) Steps 2-5 are repeated nr times but the offset of the selective pulses in the $\mathrm{SS} 2 \mathrm{M}$ block is changed so to address a different slice at each repetition.

Each spin-echo imaging acquisition is stored on a separate line of a Bruker 2D ser file. The file is then Fourier-transformed and processed in magnitude mode. The intensity of the images acquired at each step decays according to the singlet-order specific relaxation decay constant. For convenience in making comparisons, here we only report the positive projection rather than the full 2D spectrum. We point out that the spin-echo imaging acquisition can be replaced by a conventional NMR FID acquisition thus retaining spectral resolution and providing higher sensitivity, if necessary. However, since spectral resolution is unimportant in measuring $T_{\mathrm{S}}$ and since molecules displaying very long-lived spin states are nearly equivalent spin-pairs (i.e. they generate a single peak on the spectrum) we have here preferred to use a spin-echo imaging modality that gives a clearer indication of the spatial region where the signal is coming from.

In its second implementation (SSTS-v2, Fig. 1B), a train of sM2S blocks followed by a relaxation delay is run, repeatedly, to convert longitudinal into singlet order only in selected portions of the sample. At each run, the sM2S is instructed to address a different portion of the sample so that different portions are addressed at distinct times. A successive non-selective S2M converts singlet order into transverse magnetization in all slices simultaneously which is then conveniently detected through a 1D spin echo imaging sequence. The singlet prepared in each slice starts decaying immediately after preparation but the time elapsed from preparation to detection is different for the different slices so the whole array of the variable delay time can be encoded in a single spectrum. The different steps involved in SSTS-v2 can be summarized as follows:

(1) A sM2S block (Fig. 1F) creates long-lived singlet order only in a selected region of space (an axial slice perpendicular to the tube long axis). The orientation, position and thickness of this slice depend on the axis along which the gradients $G_{1}$, $\mathrm{G}_{2}$ and $\mathrm{G}_{3}$ are applied, their intensity and the offset and bandwidth of the shaped pulses (details below).

(2) During the time interval $\tau_{\mathrm{sl}}$ the singlet order created in the current and all previously addressed slices starts decaying.

(3) The sM2S is repeated nr times but, at each repetition, the offset of the selective pulses is changed so as to address a different slice. After nr repetitions, singlet order will be created in all slices but at different times to each other.

(4) A $T_{00}$ filter $^{56}$ (Fig. 1E) is applied to filter out possible sM2S by-products distinct from the singlet order.

(5) A non-selective $S 2 M^{54,55}$ (Fig. 1D) is run to convert the singlet order into transverse magnetization, simultaneously in all slices.

(6) A 1D spin echo imaging sequence is run to yield the sample profile along the $z$-axis.

The acquired echo signal is Fourier-transformed and processed in magnitude mode. The resulting spectrum contains $\mathrm{nr}$ peaks, each one directly reporting on the intensity of the singlet order evolved in one of the nr slices addressed in steps 1-3.

The gradients used for spatial selection $\left(\mathrm{G}_{1}-\mathrm{G}_{3}\right)$ have trapezoidal shape and are applied along the $z$-axis (coinciding with the direction of the $B_{0}$ field and the NMR tube long axis). All other gradients $\left(\mathrm{G}_{\mathrm{A}}-\mathrm{G}_{\mathrm{D}}\right)$ have half-sine shape and are applied along directions perpendicular to $z$. The pulse shapes are chosen to provide a good selection profile for 180 degrees pulses in the pulse sequence, their duration is adjusted to reach the desired bandwidth (BW). The strength of the $G_{1}$ gradient $\left(g_{1}\right)$ is adjusted so as to select the desired slice thickness $\mathrm{TH}$ according to

$$
\mathrm{TH}=\frac{\mathrm{BW}}{\gamma g_{1}}
$$


where $\gamma$ is the gyromagnetic ratio of the spins involved in the singlet order and $g_{1}$ is the strength of the gradient $\mathrm{G}_{1}$. The duration $\delta_{1}$ is set equal to the length of the 90 degrees selective pulse, $\mathrm{sp}_{90}$. The gradient $\mathrm{G}_{2}$ has equal but opposite strength to $\mathrm{G}_{1}$ (i.e. $g_{2}=-g_{1}$ ) whereas the duration $\delta_{2}$ is adjusted such that the total area of $\mathrm{G}_{2}$ is equal and opposite to half the area of $\mathrm{G}_{1}$ to cancel the phase distortion induced by $\mathrm{G}_{1}$ during the selective 90 degrees pulse. The gradient $\mathrm{G}_{3}$ makes the 180 degrees pulses also space selective; the intensity $g_{3}$ is set equal to $g_{1}$ while the duration $\delta_{3}$ is adjusted to match the length of the 180 degrees shaped pulse, $\mathrm{sp}_{180}$. A shaped pulse played on resonance (pulse offset $\omega_{\text {off }}=0$ ) alongside to the gradient $G_{1}$ and with bandwidth BW addresses a slice at the centre of the coil with thickness TH given in eqn (2). To target a different position along the $z$-axis it is sufficient to change $\omega_{\text {off }}$ (in both positive and negative direction) and the slice position with respect to the coil center is given by

$$
\Delta z=\frac{\omega_{\mathrm{off}}}{\gamma g_{1}}
$$

The echo delay $\tau_{\mathrm{e}}$ and the number of echo repetitions $n_{1}$ and $n_{2}$ in $\mathrm{M} 2 \mathrm{~S} / \mathrm{S} 2 \mathrm{M} / \mathrm{sM} 2 \mathrm{~S} / \mathrm{sS} 2 \mathrm{M}$ are experimentally optimized around the theoretical values reported in the caption of Fig. 1. The gradients $\mathrm{G}_{4}$ and $\mathrm{G}_{5}$ are used for the $1 \mathrm{D}$ imaging. The duration $\delta_{5}$ is set the same as the acquisition length while its intensity, $g_{5}$, is set such that the spectrum fits in the selected spectral window considering the sample and the coil dimensions. The area of the pre-phasing gradient $\mathrm{G}_{4}$ is set to be half of the area of $\mathrm{G}_{5}$ by using $g_{4}=g_{5}$ and adjusting $\delta_{4}$ accordingly. A useful equation relates the offset of the selective pulses $\left(\omega_{\text {off }}\right)$ to spectral frequency of the center of the selected slice $\left(\nu_{\mathrm{sc}}\right)$ in the 1D spin-echo profile spectrum:

$$
\omega_{\text {off }}=\nu_{\mathrm{ss}} \frac{g_{1}}{g_{5}}
$$

\section{D spin-echo images along tube main axis}

A 1D spin-echo image along the $z$-axis is acquired on sample S1 (see Materials and methods) in order to reveal the sample profile along the laboratory frame $z$-axis (aligned with the main field). This is achieved by using the pulse sequence in Fig. $1 \mathrm{H}$ with $\mathrm{G}_{4}$ and $\mathrm{G}_{5}$ applied along $z$ and with $g_{4}=g_{5}=75 \mathrm{mT} \mathrm{m}^{-1}$ ( $5 \%$ of maximum). The resulting spectrum is shown in magnitude mode in Fig. 2 (grey line). The FWHM is $\sim 54 \mathrm{kHz}$ corresponding to a sample extension along the $z$-axis of $\sim 16.9 \mathrm{~mm}$ (eqn (2)), slightly smaller than the coil dimension of $18 \mathrm{~mm}$. The small discrepancy of dimension implies that $\mathrm{B}_{1}$ inhomogeneities at the edge of the coil may slightly compromise the image quality at the edges. There is, however, a roughly $32 \mathrm{kHz}$ wide region where the spectrum intensity is high and flat, corresponding to a region of $\sim 10 \mathrm{~mm}$ where the coil performs at its best.

The red line in Fig. 2 refers to the $z$-profile image of sample S1 acquired with the sequence in Fig. $1 \mathrm{H}$ where the 1D spin-echo imaging is run on the amount of magnetization which has been firstly converted into singlet order, filtered through the $T_{00}$ filter

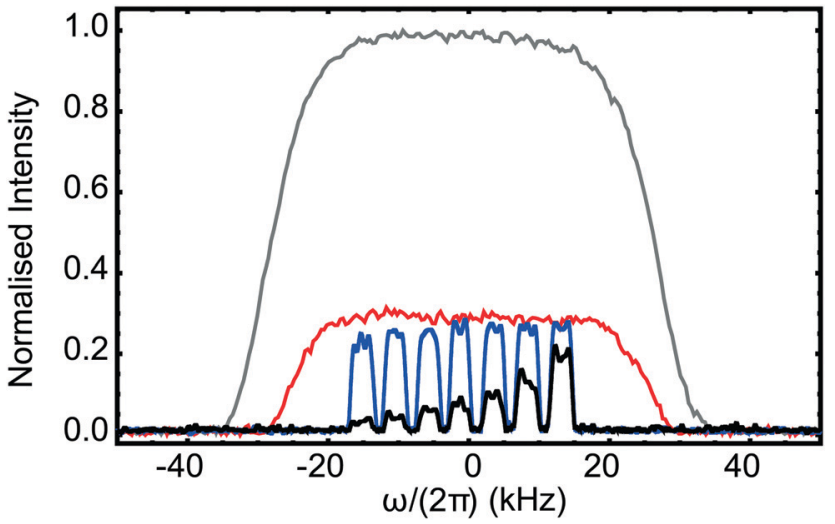

Fig. 2 1D image of sample S1 along the $z$-axis obtained using the pulse sequence in Fig. $1 \mathrm{H}$ (grey line); the pulse sequence in Fig. 11 where only the signal filtered through singlet order is acquired (red line); the pulse sequence in Fig. $1 \mathrm{~A}$ with $\mathrm{nr}=7$ and $\tau_{\mathrm{sl}}=1 \mathrm{~s}$ (blue line - positive projection across all rows); the pulse sequence in Fig. $1 \mathrm{~A}$ with $\mathrm{nr}=7$ and $\tau_{\mathrm{sl}}=60 \mathrm{~s}$ (black line - positive projection across all rows). A single transient was used to acquire each image and all other parameters are discussed in Materials and methods.

and then converted back to transverse order. Theoretically, this filtering should reduce the signal by $1 / 3$ but a more pronounced signal reduction of $\sim 2 / 3$ is experimentally observed. However, the comparison between the two profiles is not straightforward since the solvent peaks also contribute to the image taken with the pulse sequence in Fig. $1 \mathrm{H}$ while their signal is suppressed by the $T_{00}$ filter used in the sequence in Fig. 1I. The 1D spin-echo image of sample S1 produced by the SSTS-v1 pulse sequence of Fig. $1 \mathrm{~A}$ (blue line in Fig. 2) run for $\mathrm{nr}=7$ and $\tau_{\mathrm{sl}}=1 \mathrm{~s}$ contains, in its positive projection across all rows, seven well-separated peaks, one for each of the $\mathrm{nr}$ slices selected. The intensity of these peaks is only slightly smaller than the singlet-filtered 1D sample profile meaning that there are no significative losses in running spatial-selective (sS2M) rather than non-selective S2M.

The 1D spin-echo image of sample $\mathrm{S} 1$ are still produced by the SSTS-v1 pulse sequence but $\mathrm{nr}=7$ and $\tau_{\mathrm{sl}}=60 \mathrm{~s}$ (black line in Fig. 2) also shows seven well-separated peaks whose intensity decays from right to left. In this experiment, the signal from the spins located in the slice corresponding to the rightmost peak has been acquired $60 \mathrm{~s}$ after the M2S was run; the second peak from the right has been acquired $120 \mathrm{~s}$ after the M2S was run, and so on for all other peaks. The areas underneath each peak can be plotted against time and fitted to retrieve the singlet relaxation constant. Unfortunately, this fitting gives a value of $T_{\mathrm{S}}=133 \pm 15 \mathrm{~s}$ which is significantly shorter than the value of $T_{\mathrm{S}}=201 \pm 1 \mathrm{~s}$ measured with a conventional pseudo-2D experiment. This is somewhat expected and is attributable to a combination of molecular diffusion and thermal convection currents. This issue is discussed and resolved below.

\section{The effects of diffusion and thermal convection}

Right from conception, we were well aware that thermal convection and Brownian diffusion would compromise the proposed methodology. The molecule used in this work (see Materials and methods), 
has an isotropic diffusion coefficient of $6.6 \times 10^{-10} \mathrm{~m}^{2} \mathrm{~s}^{-1}$ as measured by a convection-compensated double-stimulated echo experiment. This means that it can travel an average distance of $0.25 \mathrm{~mm}$ per minute along one direction. On top of that, and much more effective, convection currents generated by temperature gradients in the sample can transport molecules across many millimeters per minute. The molecules which are in a specific location at the time the M2S is run, would be in a different position at the time the $\mathrm{SS} 2 \mathrm{M}$ is run to retrieve the signal from that specific location; this would result in an apparent loss of signal which is attributable to the physical movement of the molecule rather than to a mere nuclear spin relaxation effect. This phenomenon can be directly tracked by using the SSTS-v2 pulse sequence which can visually report on molecular motions. In this version of the experiment, in fact, the molecules in each specific location are tagged as singlet order during the SM2S initial step. During the time needed for tagging all different slices, the singlet-tagged molecules move around the sample due to the motions discussed above. The final S2M followed by spin-echo imaging acquisition reveals the positions of these molecules wherever they are at the time of detection; if the molecules have moved out of the region where they were at the time of tagging, their final position will be revealed at the time of detection, i.e. the peaks in the image which correspond to the signal from the spins tagged in a given location will broaden proportionally to the extent of molecular motion. This is exactly what happened for sample $\mathrm{S} 1$ when the pulse sequence in Fig. $1 \mathrm{~B}$ was run at $\mathrm{nr}=7$ and $\tau_{\mathrm{sl}}=1 \mathrm{~s}$ or $30 \mathrm{~s}$ as shown in Fig. 3 . In this figure, the rightmost peak corresponds to the signal from the slice selected by the first occurrence of the sM2S, the second peak from the right corresponds to the signal from the slice selected by the second occurrence of the sM2S and so on for all other peaks. It becomes clear from these experiments that thermal convection is deleterious for the quantitative determination of $T_{\mathrm{S}}$ in these experiments; even for $\tau_{\mathrm{sl}}=1 \mathrm{~s}$, the peaks start to merge meaning that molecules have travelled the $0.5 \mathrm{~mm}$ left between two consecutive slices. This motion is almost entirely due to thermal convection since Brownian diffusion would only account for micrometric displacements in such a short time. For $\tau_{\mathrm{sl}}=30 \mathrm{~s}$ (Fig. $3 \mathrm{~B}$ ), the molecules originally tagged in a given slice have spread across the whole sample as indicated by the almost complete merging of all peaks.

Incidentally, the peaks produced by the pulse sequence SSTS-v1 remain of a fixed width irrespective of the value of $\tau_{\mathrm{sl}}$ (compare blue and black lines in Fig. 2) since in this version of the experiment the time between the selective tagging of the molecules in a specific location occurs immediately before detection so that no displacement is actually visible. This does not mean that SSTS-v1 is actually immune to molecular motions since this is always going on in background, manifesting itself as a limiting factor on the accuracy on the $T_{\mathrm{S}}$ measurements.

The extent of thermal convection depends on many factors including temperature difference across the sample, viscosity of the solvent, tube diameter and more, ${ }^{57}$ so its effects will manifest differently in different samples. Even if we could find a way to reduce convection to zero by, for example, a perfect
A

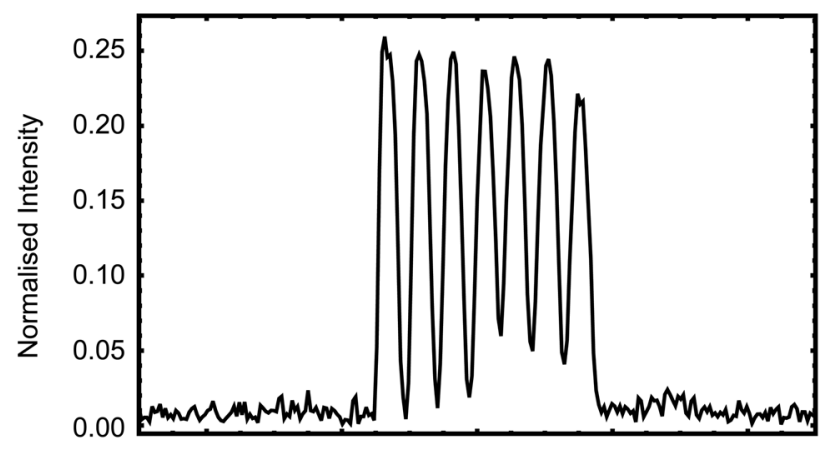

B

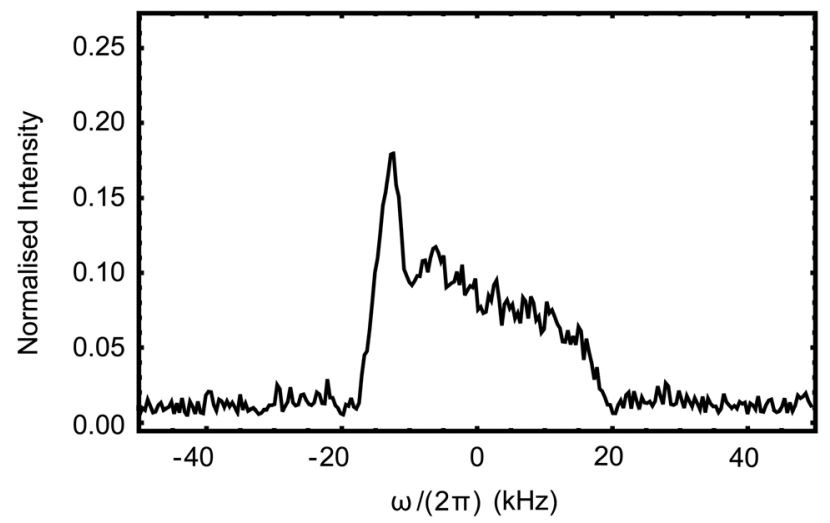

Fig. 3 1D spin echo images of sample S1 obtained with the sequence in Fig. $1 \mathrm{~B}$ for $\mathrm{ns}=1, \mathrm{nr}=7$ and $\tau_{\mathrm{sl}}=1 \mathrm{~s}(\mathrm{~A})$ and $\tau_{\mathrm{sl}}=30 \mathrm{~s}(\mathrm{~B})$. The intensity is normalized to the one of Fig. 2.

control of the sample temperate, molecules would still move because of Brownian diffusion. Therefore, during the long $\tau_{\mathrm{sl}}$ required to measure minutes long $T_{\mathrm{S}}$, molecules would still be excited while they are in a slice but are detected when they have moved to another one, thus compromising the whole experiment. The only way to make this experiment quantitative is to physically confine molecules within the selected slices. This is equivalent to the use of Shigemi tubes to confine all spins within the detecting coil in experiments where molecular motions can bring molecules outside of the coil at the time of detection.

\section{Limiting molecular motions using compartmentation}

To confine molecular motions in our experiments and thus make those quantitative, we propose to use a tube insert with a number of physically separated compartments (7 in our case) and set up the offsets of all selective pulses in the SSTS pulse sequences such that the center of each selected slice coincides with the center of these compartments. A prototype of this insert is discussed in Materials and methods and fits a conventional $10 \mathrm{~mm}$ medium-wall NMR tube. The insert has seven $1 \mathrm{~mm}$ thick compartments separated by $0.5 \mathrm{~mm}$ thick walls and is machined from a polyoxymethylene (POM) rod. The choice of the material is simply based on its chemical compatibility with most organic solvents and its mechanical properties in relation 
to the machining process. Since the insert OD is slightly smaller than the tube ID the compartments are easily filled as the solution is poured from the top making it quite compatible with dissolution-DNP experiments, for example. Air bubbles possibly trapped in the compartments are easily removed by a quick shake. For hyperpolarization experiments, where there is plenty of signal, the chambers in the insert can be fabricated thinner and arranged parallel to the tube main axis so as to make them easier to be filled with the sample coming out from the polariser. The 1D spin-echo image and the singlet-filtered 1D spin-echo image of a $0.5 \mathrm{M}$ solution of EPM dissolved in acetone$\mathrm{d}_{6}$ and contained in a LPV $10 \mathrm{~mm}$ OD medium-wall tube also containing the POM insert (sample S2) are shown in Fig. 4A and B. The signal from the molecules contained in each compartment appears in the form of well-separated peaks in the image. The small differences in the width and height of these peaks are due to imperfections in the machining which we can take into account as explained below. The small bumps at each side are due to the small amount of sample enclosed between the glass walls and the insert at the edges of the coil. Our coil is $18 \mathrm{~mm}$ long and therefore extends for about $4 \mathrm{~mm}$ each side of the compartmented region of the insert, which is just $10 \mathrm{~mm}$ long.

Fig. 4C displays the positive projection of the $2 \mathrm{D}$ spectrum resulting from the pulse sequence SSTS-v1 run with $\mathrm{nr}=7$ and $\tau_{\mathrm{sl}}=1 \mathrm{~s}$ on sample $\mathrm{S} 2$ where seven $1.2 \mathrm{~mm}$ thick slices are selected using $g_{1}=-g_{2}=g_{3}=420 \mathrm{mT} \mathrm{m}^{-1}$ and the offset of the selective pulses carefully chosen so as to center the selected slices with the center of the compartments in the POM insert easily done using eqn (4). The thickness of these slices $(1.2 \mathrm{~mm})$ was deliberately chosen to be slightly bigger than the POM compartments $(1 \mathrm{~mm})$ to ensure a uniform excitation of the sample inside each compartment. The peaks are again wellseparated, and the intensity pattern follows the one of the sample $z$-profiles in Fig. 4A. The spectrum in Fig. $4 \mathrm{D}$ is the

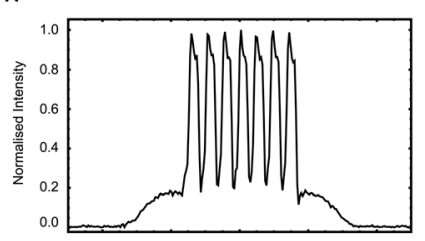

C

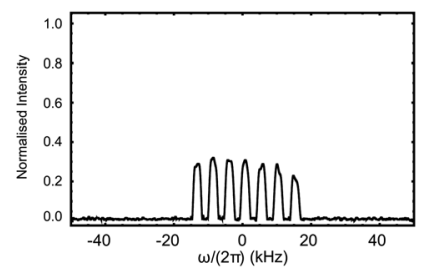

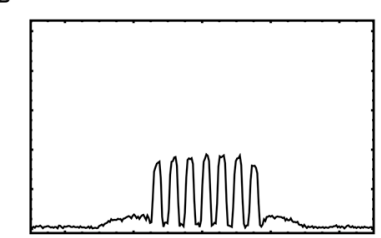

D

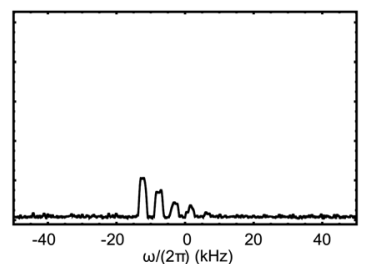

Fig. 4 1D spin-echo image of S2 obtained using the sequence in Fig. $1 \mathrm{H}$ (A); 1D singlet-filtered spin-echo image of $\$ 2$ obtained using the sequence in Fig. 11 (B). Positive projections across all rows or the $2 \mathrm{D}$ spectrum resulting from the SSTS-v1 sequence in Fig. $1 \mathrm{~A}$ run with $\mathrm{nr}=7$ and $\tau_{\mathrm{sl}}=1 \mathrm{~s}$ (C) and $\tau_{\mathrm{sl}}=60 \mathrm{~s}$ (D). The intensity is normalized across all panels such that the highest peak in panel $A$ is 1. positive projection of the $2 \mathrm{D}$ spectrum resulting from the pulse sequence SSTS-v1 run with $\mathrm{nr}=7$ and $\tau_{\mathrm{sl}}=60 \mathrm{~s}$ on sample S2.

The peaks remain well defined for the reasons discussed above but their intensities decay according to the time the corresponding slice has been selected, with the leftmost peak being selected at the first $\mathrm{SS} 2 \mathrm{M}$ occurrence and the rightmost peak being selected at its last.

\section{Singlet order decay constant quantification}

To measure $T_{\mathrm{S}}$ using the SSTS-v1 method we simply calculate the area under each peak in the spectrum with $\tau_{\mathrm{sl}}=60 \mathrm{~s}$ (Fig. 4D). Normalizing this to the area of the corresponding peak in the singlet-filtered 1D spin-echo image (Fig. 4B), the possible differences in volume between the compartments due to imprecise mechanical machining of the insert and/or differences in $\mathrm{B}_{1}$ homogeneity at the edges of the sample can be accounted for.

The normalized area of the seven peaks is plotted versus time in Fig. 5 and fitted to a mono-exponential decay function to yield a $T_{\mathrm{S}}=134 \pm 11 \mathrm{~s}$ which is in good agreement with a value of $142 \pm 3$ obtained using the conventional pseudo-2D method.

The total duration of the SSTS-v1 experiment in Fig. 5 was $\sim \mathrm{nr} \times \tau_{\mathrm{sl}}=420 \mathrm{~s}$ to be compared with the $1852 \mathrm{~s}$ needed by the conventional pseudo-2D measurements and as calculated using eqn (1) using the experimental value of $T_{1}$ of $8.2 \mathrm{~s}$ and the same linear array of 7 points spanning from 60 to $420 \mathrm{~s}$. This corresponds to a time saving factor of 4.4. The saving factor can change depending on the way the variable time array is arranged but the absolute time saving becomes more and more significant when the $T_{1}$ and $T_{\mathrm{S}}$ of the sample become longer and longer. Recalling the example in the Introduction, a molecule with an estimated $T_{\mathrm{S}}$ of $60 \mathrm{~min}$ and a $T_{1}$ of $30 \mathrm{~s}$ would require $\sim 11 \mathrm{~h}$ per scan to measure $T_{\mathrm{S}}$ in a conventional pseudo-2D mode whereas our SSTS method would achieve the same goal in just $3 \mathrm{~h}$ per scan.

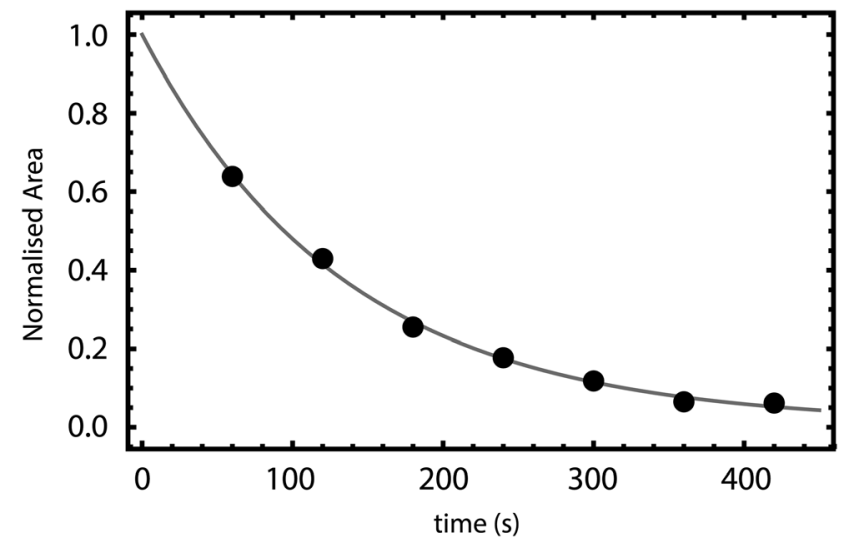

Fig. 5 Filled circles are the experimental area under the peaks in the spectrum of Fig. 4D. Each area has been normalized to the area of the corresponding peak in Fig. 4 and, successively, all points have been normalised so to have 1 at time 0 . The time axis is obtained as an integer increment of $\tau_{\mathrm{sl}}$ from 1 to 7 . The grey line is the best fit to a monoexponential decay. 
A

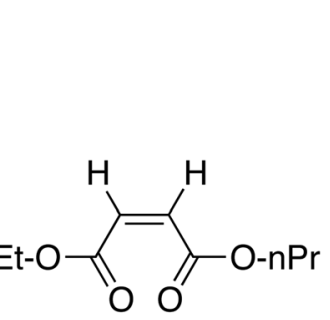

B

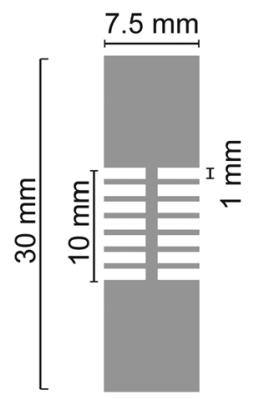

Fig. 6 Structure of molecule used and geometry of sample insets. (A) Molecular structure of ethyl- $d_{5}$ (propyl- $d_{7}$ ) maleate (EPM); (B) details of the POM insert used in sample S2.

\section{Materials and methods}

\section{Equipment}

All experiments have been run on a $7.04 \mathrm{~T}$ wide bore magnet coupled to a Bruker Avance III console fitted with a 3-axis gradient system for micro-imaging experiments, able to deliver a maximum field gradient of $1.5 \mathrm{~T} \mathrm{~m}^{-1}$, and a Bruker MICWB40 microimaging probe equipped with a ${ }^{1} \mathrm{H} /{ }^{13} \mathrm{C} 10 \mathrm{~mm}$ resonator.

\section{Samples}

All experiments have been run on samples containing a $\sim 0.5 \mathrm{M}$ solution of ethyl- $\mathrm{d}_{5}$ (propyl- $\mathrm{d}_{7}$ ) maleate (EPM, Fig. 6A) dissolved in ethanol- $\mathrm{d}_{6}$ and degassed by bubbling oxygen-free $\mathrm{N}_{2}$ gas.

The molecule of EPM was synthetized in house according to reported procedures. ${ }^{43}$ For experiments referring to sample S1, the solution was contained in $10 \mathrm{~mm}$ medium-wall LPV NMR tube attached to a large J-Young valve that allows a $7.5 \mathrm{~mm}$ OD object to comfortably pass through its neck. For experiments referring to sample $\mathrm{S} 2$, the very same tube used for sample S1 was opened and a plastic structure inserted. This structure, machined from a polyoxymethylene rod with the dimensions specified in Fig. 6B, was designed in order to confine molecular motions within 7 parallel $1 \mathrm{~mm}$-thick compartments spaced by six $0.5 \mathrm{~mm}$-thick walls.

The $T_{1}$ decay constant of samples $\mathrm{S} 1$ and $\mathrm{S} 2$ have been measured by a conventional saturation recovery experiment and found to be $8.2 \pm 0.8 \mathrm{~s}$. The $T_{\mathrm{S}}$ for those two samples, measured with the conventional pseudo-2D mode, were found to be $201 \pm$ $1 \mathrm{~s}$ for S1 and $143 \pm 3 \mathrm{~s}$ for S2. The discrepancy between two samples is attributable to the likely higher level of $\mathrm{O}_{2}$ present in the sample $\mathrm{S} 2$ after the opening and placing of the plastic insert.

\section{Pulse sequence parameters}

Selective excitation and refocusing pulses occurring in the pulse sequences of Fig. 1 were designed using the Shinnar-Le Roux (SLR) algorithm using an equiripple filter and a timebandwidth product of $6 .^{58}$ These pulses were used with duration $\mathrm{sp}_{90}=590 \mu \mathrm{s}$ and $\mathrm{sp}_{180}=635 \mu \mathrm{s}$ for excitation and refocusing, respectively, corresponding to a bandwidth $\mathrm{BW}=$ $9 \mathrm{kHz}$. The strength of $\mathrm{G}_{4}$ and $\mathrm{G}_{5}$ gradients was kept constant at $75 \mathrm{mT} \mathrm{m}^{-1}$ (5\% of maximum available) throughout all

experiments, and their duration was $2.56 \mathrm{~ms}$ (matched to a SW of $100 \mathrm{kHz}$ and 512 points in the time domain) and $1.28 \mathrm{~ms}$, respectively. The absolute strengths $g_{1}, g_{2}$ and $g_{3}$ were set to $180 \mathrm{mT} \mathrm{m}^{-1}$ (12\% of maximum available). From eqn (2), these gradients excite a $1.2 \mathrm{~mm}$ thick slice. The optimized values of the echo delay and echo repetitions in the sM2S and S2M blocks were $\tau_{\mathrm{e}}=41.8 \mathrm{~ms}, n_{1}=18$ and $n_{2}=9$. Seven slices were selected $(\mathrm{nr}=7)$ and the offsets of the selective pulses to select the 7 slices were chosen so as to coincide with the center of the compartments in the POM insert.

\section{Conclusions}

The methodology introduced in this paper allows single-scan measurements of the decay rate of singlet order. The method offers a significant saving factor in experimental time which translates into important and substantial absolute time saving when measuring samples with long $T_{1}$ and $T_{\mathrm{S}}$ values. Even more important is the fact that this is a single-scan method and therefore marries well with single-scan hyperpolarization techniques such as dissolutionDNP. Measuring $T_{\mathrm{S}}$ lifetimes of hyperpolarized samples is otherwise very challenging, requiring multiple repetitions of the hyperpolarization procedures. Not only does this repetition have obvious implications on costs and time but, perhaps more importantly, causes irreproducibility associated with the difficulty of producing batches of identical polarization repeatedly.

As in all other spatial-encoding based techniques, our method requires a trade-off between the number of points acquired simultaneously and the reduction in signal-to-noise due to the fact that the signal comes from a restricted portion of the sample. However, this should not be anything to worry about in the presence of hyperpolarization which is where we feel the technique expresses its best potential.

We believe that this methodology can play a fundamental role in the quest for singlet-bearing molecules displaying long lifetimes. Often in this field, we are required to design and synthesise doubly labelled molecules guided by scientific reasoning and/or simulations of the possible value of $T_{\mathrm{S}}$. This is a costly and time-consuming procedure and may well end up revealing that the molecule is not suitable for the desired purpose. The advances reported here provided by our new methodology in combination with a hyperpolarization method, could be used to measure $T_{\mathrm{S}}$ in naturally abundant (or, at least, singly labelled) two-spin systems thus allowing evaluation of the suitability of a molecule, for any given application, before investing time and money on isotopically enriched syntheses. Investigation of hyperpolarized substrates will be carried out when the worldwide health emergency has passed, and interactions with other groups equipped with such technology becomes possible again.

\section{Author contributions}

G. P. and J. N. D. devised the research and ran experiments; G. M. and C. N. ran experiments and processed the data; L. J. B. synthetized the molecules. G. P. wrote the paper. 


\section{Conflicts of interest}

There are no conflicts to declare.

\section{Acknowledgements}

The authors thank Stuart J. Elliott, Ilya Kuprov and Ahmed J. M. Allami for fruitful discussions. This research was supported by EPSRC (UK) grant no. EP/P005187/1.

\section{Notes and references}

1 Long-lived Nuclear Spin Order: Theory and Applications, ed. G. Pileio, Royal Society of Chemistry, London, 2020.

2 C. Bengs, M. Sabba, A. Jerschow and M. H. Levitt, Phys. Chem. Chem. Phys., 2020, 22, 9703-9712.

3 B. A. Rodin, C. Bengs, A. S. Kiryutin, K. F. Sheberstov, L. J. Brown, R. C. D. Brown, A. V. Yurkovskaya, K. L. Ivanov and M. H. Levitt, J. Chem. Phys., 2020, 152, 164201.

4 S. Mamone, N. Rezaei-Ghaleh, F. Opazo, C. Griesinger and S. Glöggler, Sci. Adv., 2020, 6, eaaz1955.

5 C. Bengs, L. Dagys and M. H. Levitt, J. Magn. Reson., 2020, 321, 106850.

6 B. A. Rodin, K. F. Sheberstov, A. S. Kiryutin, L. J. Brown, R. C. D. Brown, M. Sabba, M. H. Levitt, A. V. Yurkovskaya and K. L. Ivanov, J. Chem. Phys., 2019, 151, 234203.

7 B. A. Rodin, A. S. Kiryutin, A. V. Yurkovskaya, K. L. Ivanov, S. Yamamoto, K. Sato and T. Takui, J. Magn. Reson., 2018, 291, 14-22.

8 G. Pileio, Prog. Nucl. Magn. Reson. Spectrosc., 2017, 98-99, 1-19.

9 G. Stevanato, J. Eills, C. Bengs and G. Pileio, J. Magn. Res., 2017, 277, 169-178.

10 J. Eills, G. Stevanato, C. Bengs, S. Glöggler, S. J. Elliott, J. Alonso-Valdesueiro, G. Pileio and M. H. Levitt, J. Magn. Res., 2017, 274, 163-172.

11 A. S. Kiryutin, A. N. Pravdivtsev, A. V. Yurkovskaya, H. M. Vieth and K. L. Ivanov, J. Phys. Chem. B, 2016, 120, 11978-11986.

12 Z. Zhou, K. Claytor, W. S. Warren and T. Theis, J. Magn. Reson., 2016, 263, 108-115.

13 K. Claytor, T. Theis, Y. Feng and W. Warren, J. Magn. Reson., 2014, 239, 81-86.

14 K. Claytor, T. Theis, Y. Feng, J. Yu, D. Gooden and W. S. Warren, J. Am. Chem. Soc., 2014, 136, 15118-15121.

15 A. S. Kiryutin, K. L. Ivanov, A. V. Yurkovskaya, H.-M. Vieth and N. N. Lukzen, Phys. Chem. Chem. Phys., 2013, 15, 14248-14255.

16 S. J. DeVience, R. L. Walsworth and M. S. Rosen, Phys. Rev. Lett., 2013, 111, 5.

17 R. Sarkar, P. Ahuja, D. Moskau, P. R. Vasos and G. Bodenhausen, ChemPhysChem, 2007, 8, 2652-2656.

18 R. Buratto, D. Mammoli, E. Chiarparin, G. Williams and G. Bodenhausen, Angew. Chem., Int. Ed., 2014, 53, 11376-11380.

19 N. Salvi, R. Buratto, A. Bornet, S. Ulzega, I. Rentero Rebollo, A. Angelini, C. Heinis and G. Bodenhausen, J. Am. Chem. Soc., 2012, 134, 11076-11079.
20 M. C. Tourell, I.-A. Pop, L. J. Brown, R. C. D. Brown and G. Pileio, Phys. Chem. Chem. Phys., 2018, 20, 13705-13713.

21 G. Pileio and S. Ostrowska, J. Magn. Reson., 2017, 285, 1-7.

22 G. Pileio, J.-N. Dumez, I.-A. Pop, J. T. Hill-Cousins and R. C. D. Brown, J. Magn. Reson., 2015, 252, 130-134.

23 J. N. Dumez, J. T. Hill-Cousins, R. C. D. Brown and G. Pileio, J. Magn. Reson., 2014, 246, 27-30.

24 P. Ahuja, R. Sarkar, P. R. Vasos and G. Bodenhausen, J. Am. Chem. Soc., 2009, 131, 7498-7499.

25 S. Cavadini, J. Dittmer, S. Antonijevic and G. Bodenhausen, J. Am. Chem. Soc., 2005, 127, 15744-15748.

26 P. Saul, S. Mamone and S. Glöggler, Chem. Sci., 2019, 10, 413-417.

27 S. Yang, J. McCormick, S. Mamone, L. S. Bouchard and S. Glöggler, Angew. Chem., Int. Ed., 2019, 58, 2879-2883.

28 C. P. N. Tanner, J. R. Lindale, S. L. Eriksson, Z. Zhou, J. F. P. Colell, T. Theis and W. S. Warren, J. Chem. Phys., 2019, 151, 044201.

29 S. Mamone and S. Glöggler, Phys. Chem. Chem. Phys., 2018, 20, 22463-22467.

30 T. Theis, G. X. Ortiz, A. W. Logan, K. E. Claytor, Y. Feng, W. P. Huhn, V. Blum, S. J. Malcolmson, E. Y. Chekmenev, Q. Wang and W. S. Warren, Sci. Adv., 2016, 2, e1501438.

31 J.-N. Dumez, P. Håkansson, S. Mamone, B. Meier, G. Stevanato, J. T. Hill-Cousins, S. S. Roy, R. C. D. Brown, G. Pileio and M. H. Levitt, J. Chem. Phys., 2015, 142, 044506.

32 Y. Feng, T. Theis, X. Liang, Q. Wang, P. Zhou and W. S. Warren, J. Am. Chem. Soc., 2013, 135, 9632-9635.

33 S. J. DeVience, R. L. Walsworth and M. S. Rosen, NMR Biomed., 2013, 26, 1204-1212.

34 Y. Zhang, X. Duan, P. C. Soon, V. Sychrovský, J. W. Canary and A. Jerschow, ChemPhysChem, 2016, 17, 2967-2971.

35 Y. Zhang, K. Basu, J. W. Canary and A. Jerschow, Phys. Chem. Chem. Phys., 2015, 17, 24370-24375.

36 Y. N. Zhang, P. C. Soon, A. Jerschow and J. W. Canary, Angew. Chem., Int. Ed., 2014, 53, 3396-3399.

37 C. R. Bowers and D. P. Weitekamp, J. Am. Chem. Soc., 1987, 109, 5541-5542.

38 R. W. Adams, J. A. Aguilar, K. D. Atkinson, M. J. Cowley, P. I. Elliott, S. B. Duckett, G. G. Green, I. G. Khazal, J. LópezSerrano and D. C. Williamson, Science, 2009, 323, 1708-1711.

39 J. H. Ardenkjaer-Larsen, B. Fridlund, A. Gram, G. Hansson, L. Hansson, M. H. Lerche, R. Servin, M. Thaning and K. Golman, Proc. Natl. Acad. Sci. U. S. A., 2003, 100, 10158-10163.

40 A. N. Pravdivtsev, F. D. Sönnichsen and J.-B. Hövener, PLoS One, 2020, 15, e0239982.

41 G. Pileio, Prog. Nucl. Magn. Reson. Spectrosc., 2010, 56, 217-231.

42 G. Stevanato, J. T. Hill-Cousins, P. Håkansson, S. S. Roy, L. J. Brown, R. C. D. Brown, G. Pileio and M. H. Levitt, Angew. Chem., Int. Ed., 2015, 54, 3740-3743.

43 L. J. Brown, in Long-lived nuclear spin order: theory and applications, ed. G. Pileio, Royal Society of Chemistry, London, 2020, ch. 4. 
44 D. Graafen, M. B. Franzoni, L. M. Schreiber, H. W. Spiess and K. Münnemann, J. Magn. Res., 2016, 262, 68-72.

45 G. Pileio, S. Bowen, C. Laustsen, M. C. D. Tayler, J. T. HillCousins, L. J. Brown, R. C. D. Brown, J. H. ArdenkjaerLarsen and M. H. Levitt, J. Am. Chem. Soc., 2013, 135, 5084-5088.

46 L. Frydman, T. Scherf and A. Lupulescu, Proc. Natl. Acad. Sci. U. S. A., 2002, 99, 15858-15862.

47 N. M. Loening, M. J. Thrippleton, J. Keeler and R. G. Griffin, J. Magn. Reson., 2003, 164, 321-328.

48 M. J. Thrippleton, N. M. Loening and J. Keeler, Magn. Reson. Chem., 2003, 41, 441-447.

49 L. Frydman and D. Blazina, Nat. Phys., 2007, 3, 415-419.

50 J.-N. Dumez, J. Milani, B. Vuichoud, A. Bornet, J. LalandeMartin, I. Tea, M. Yon, M. Maucourt, C. Deborde, A. Moing, L. Frydman, G. Bodenhausen, S. Jannin and P. Giraudeau, Analyst, 2015, 140, 5860-5863.
51 L. Guduff, D. Kurzbach, C. van Heijenoort, D. Abergel and J.-N. Dumez, Chem. - Eur. J., 2017, 23, 16722-16727.

52 S. Ahola, V. V. Zhivonitko, O. Mankinen, G. Zhang, A. M. Kantola, H.-Y. Chen, C. Hilty, I. V. Koptyug and V.-V. Telkki, Nat. Commun., 2015, 6, 8363.

53 P. Giraudeau, Y. Shrot and L. Frydman, J. Am. Chem. Soc., 2009, 131, 13902-13903.

54 M. C. D. Tayler and M. H. Levitt, Phys. Chem. Chem. Phys., 2011, 13, 5556-5560.

55 G. Pileio, M. Carravetta and M. H. Levitt, Proc. Natl. Acad. Sci. U. S. A., 2010, 107, 17135-17139.

56 M. C. D. Tayler, in Long-lived nuclear spin order: theory and applications, ed. G. Pileio, Royal Society of Chemistry, London, 2020, ch. 10.

57 I. Swan, M. Reid, P. W. Howe, M. A. Connell, M. Nilsson, M. A. Moore and G. A. Morris, J. Magn. Reson., 2015, 252, 120-129.

58 J. Pauly, P. Le Roux, D. Nishimura and A. Macovski, IEEE Trans. Med. Imag., 1991, 10, 53-65. 